\title{
TESTING LIVENESS PROPERTIES
}

\section{Approximating Liveness Properties by Safety Properties}

\author{
Ulrich Ultes-Nitsche and Simon St James \\ University of Southampton \\ Department of Electronics and Computer Science \\ Southampton, SO17 1BJ, United Kingdom \\ \{uun,spstj99r\}@ecs.soton.ac.uk
}

\begin{abstract}
Satisfaction within fairness establishes an abstract notion of truth under fairness in linear-time verification. It can be argued that the idea of an implementation meeting the intersection of the system behaviour and the requirement specification forms a desirable basis for testing systems. Unfortunately this intersection of the behaviours of the system and the requirements specification is not accessible directly and cannot be computed practically. It is, however, possible to derive some information of this intersection from a high-level (abstract) specifation of the system. We will present in this paper an abstraction-based approach that will enable us to derive some test cases, even for liveness properties.
\end{abstract}

Keywords: Test case generation, satisfaction within fairness, liveness properties, behaviour abstraction.

\section{INTRODUCTION}

In [12], the concept of satisfaction within fairness is presented and adopted to testing systems. The basic idea is to test a system not against requirement specifications (properties) on their own but against the intersection of the requirments specification with the behaviour of the system. A similar observation was made by Petrenko [10] at the same conference (the observation was made in his presentation). Such an approach is more suited towards verification than towards testing. The reason being the accessability of the above mentioned intersection. We simply have no direct access to an exhaustive system behaviour description of the implemented system. However, we normally will have a high-level system specification on which the implementation is based upon.

We will give in this paper a definition of a safety property that approximates a liveness property. Using this safety approximation will enable us to test for violation of liveness properties in principle. The established definition will be 
similar to the notion of satisfaction within fairness in verification $[6 ; 7 ; 12]$. In order to overcome practical limitation of the introduced concepts, we will adopt an abstraction result for satisfaction within fairness that will make the results of this paper more applicable.

\section{LINEAR-TIME VERIFICATION}

We review linear satisfaction of properties very briefly in this section. Let $A$ be the finite set of actions that a system can perform. By $A^{\omega}$ we denote the set of all infinitely long sequences of actions in $A$. A computation $c$ of the system is an element of $A^{\omega}$, i.e. $c=a_{1} a_{2} a_{3} \ldots$ such that all $a_{i}$ are in $A$. The behaviour $B$ of the system is then a subset of $A^{\omega}$. ${ }^{1}$ A property $P$, from an abstract point of view, is itself a subset of $A^{\omega}$, representing all correct behaviours with respect to the intuitive meaning of the property [1]. Hence the system, represented by its behaviour $B$, satisfies property $P$ (written: $B \vDash P$ ) if and only if all computations in $B$ are also in $P$. This linear-time satisfaction of properties is defined by

$$
B \vDash P \Longleftrightarrow B \subseteq P .
$$

\section{EXHAUSTIVE TESTING}

In contrast to the satisfaction relation for linear-time temporal verification, when testing a system with respect to a property $P(P$, in this context, can be called a requirement specification), we consider finite behaviours: We can only observe a system for a finite amount of time, during which we only see a prefix of its (potentially) infinite behaviour. Let us define formally the relation that describes testing a system exhaustively:

Let $A^{*}$ denote the set of all finitely long sequences of actions in $A$. For a subset $S$ of $A^{\omega}$ let $\operatorname{pre}(S)$ denote the set $\left\{w \in \mathcal{A}^{*} \mid \exists x \in \mathcal{A}^{\omega}: w x \in S\right\}$ of finite prefixes of behaviours in $S$. For an element $s$ of $A^{\omega}$, let pre $(s)=$ $\operatorname{pre}(\{s\}) . B$ satisfies $P$ with respect to exhaustive testing, written $B \vdash P$ in this paper, if and only if

$$
\operatorname{pre}(B) \subseteq \operatorname{pre}(P) .
$$

Typically, properties are classified into two groups, which reflect either absence of errors or satisfactory progress. These two property classes are called safety and liveness properties respectively [1]:

$P$ is a liveness property if and only if

$$
\forall w \in \mathcal{A}^{*}: \exists x \in \mathcal{A}^{\omega}: w x \vDash P .
$$

\footnotetext{
${ }^{1}$ In terms of formal language theory, $c$ is an $\omega$-word and $B$ is an $\omega$-language on the alphabet $A$.
} 
$P$ is a safety property if and only if

$$
\forall x \in \mathcal{A}^{\omega}:\left(x \not \forall P \Rightarrow \exists w \in \operatorname{pre}(x): \forall z \in \mathcal{A}^{\omega}: w z \not \forall P\right) .
$$

Note that any property is the intersection of a safety and a liveness property [1]. Also note that $P$ is a liveness property if and only if $\operatorname{pre}(P)=A^{*}$ (recall that $P$ is a subset of $\left.A^{\omega}\right)$ [2]. Therefore $\operatorname{pre}(B)$ is always a subset of $\operatorname{pre}(P)$ for a liveness property $P$. In other words: We cannot test liveness properties, because they are always trivially satisfied (wrt. exhaustive testing).

In the case of safety properties, however, exhaustive testing is as precise as linear-time verification:

Lemma $1 P$ is a safety property if and only if $B \vdash P \Leftrightarrow \quad B \vDash P$, for all $B$.

Proof "if:" We assume $B \vdash P \Rightarrow B \vDash P$ and take the counterpositive: $B \not P \Rightarrow B \forall P$, for all $B$. Let $x \in A^{\omega}$ such that $x \not \forall P$. Then, by the above assumption, $x \forall P$. Let $w \in \operatorname{pre}(x)$ such that $w \notin \operatorname{pre}(P)$. Then for all $z \in A^{\omega}, w z \not P$. As $x$ was chosen arbitrarily, $P$ is a safety property.

"only if:" Let $P$ be a safety property. Since $B \vDash P$ always implies $B \vdash P$, we only have to show $B \vdash P \Rightarrow B \vDash P$. We prove the counterpositive version: $B \not \forall P \Rightarrow B \forall P$. Assume $B \not P P$. Then there must be an $x \in B$ such that $x \not \forall P$. Because $P$ is a safety property, there must be a prefix $w$ of $x$ such that for all $z \in A^{\omega}, w z \not \forall$. Therefore $w \notin$ pre $(P)$. Because $w \in \operatorname{pre}(B), w e$ have $B \forall P$, completing the proof.

\section{APPROXIMATING LIVENESS PROPERTIES BY SAFETY PROPERTIES}

We are going to define to a behaviour $B$ and a property $P$ a safety property $S_{B P}$. Testing $S_{B P}$ exhaustively will establish an approximation to the lineartime satisfaction of $P$ by $B$. Note that $P$ can be any property, e.g. a liveness property.

First, we need a dual operation to constructing prefix sets: the Eilenberglimit. Let $L$ be a subset of $A^{*}$. The Eilenberg-limit $\lim (L)[3 ; 11]$ is defined by

$$
\lim (L)=\left\{x \in \mathcal{A}^{\omega}|| \operatorname{pre}(x) \cap L \mid=\infty\right\} .
$$

We define the safety property to $B$ and $P$ by

$$
S_{B P}=\lim (\operatorname{pre}(B \cap P)) .
$$

First we prove that $S_{B P}$ is indeed a safety property:

Lemma $2 S_{B} P$ is a safety property.

Proof Let $x \in A^{\omega}$. If $x \not S_{B P}$, then there must be a prefix $w \in \operatorname{pre}(x)$ that is not in $\operatorname{pre}(B \cap P)$ (otherwise $x \vDash S_{B} P$ ). Then, for all $z \in A^{\omega}$, 
$w z \notin \lim (\operatorname{pre}(B \cap P))$ and thus $w z \not \forall S_{B P}$. Hence $S_{B P}$ is a safety property.

Note that $\operatorname{pre}(\lim (\operatorname{pre}(B \cap P)))=\operatorname{pre}(B \cap P)$. Hence

$$
B \vdash S_{B P} \Longleftrightarrow \operatorname{pre}(B) \subseteq \operatorname{pre}(B \cap P) .
$$

This relation also occurs in the context of linear-time properties with an abstraction notion of fairness [7;12]. We use the following result to interpret the definition of $S_{B P}$ with respect to exhaustive testing:

Lemma 3 Let $B \subseteq A^{\omega}$ and $P \subseteq A^{\omega}$ be the behaviour of a system and the property it should meet respectively. Then $B \vdash S_{B} P$ if and only if $B \cap P$ is a dense set in $B$ [7; 12].

We use in this lemma the following definition of a metric space (known as the Cantor topology) (for topological notions see [4]):

Let common $(a, b)$ designate the longest common prefix of two computations $a$ and $b$ in $A^{\omega}$. We define the metric $d(a, b)$ by

$$
\begin{gathered}
\forall a, b \in \mathcal{A}^{\omega}, a \neq b: d(a, b)=\frac{1}{|\operatorname{common}(a, b)|+1} \\
\forall b \in \mathcal{A}^{\omega}: d(b, b)=0 .
\end{gathered}
$$

How can we interpret the obtained results? Lemma 3 tells us that testing safety property $S_{B P}$ exhaustively is equivalent to checking whether $B \cap P$ is a dense set in $B$. However, in topological terms, $B \cap P$ being a dense set in $B$ is an approximation of $B \subseteq B \cap P$ and therefore an approximation of linear time satisfaction $B \vDash P$ : A system whose behaviour satisfies that $B \cap P$ is a dense set in $B$, but not that $B \vDash P$, must be observed for an infinite amount of time in order to detect $B \not \forall P[12]$. This distinction is therefore practically irrelevant, since systems cannot be observed for an infinite amount of time. Because $S_{B P}$ is a safety property, we can indeed test it. As $P$ can even be a liveness property, we have found a way to check the (approximate) satisfaction of a (liveness) property by testing a related safety property exhaustively.

Lemma 3, establishing a very interesting theoretical result about the testability of liveness properties, is unfortunately not too practical without additional effort: As we need access to $B \cap P$ in the definition of $S_{B P}$, we must be able to construct the intersection of the implementation of a system $(B)$ and the property $(P)$. But the implementation will in practice not be accessible in such a way that this intersection could be constructed.

Having mentioned above that the relation $\operatorname{pre}(B) \subseteq \operatorname{pre}(B \cap P)$ is a satisfaction relation in verification under fairness constraint (called satisfaction within fairness), we can use an abstraction result for this relation that will allow us to use the results of this section at least partly (in general we will lose some information, but not all). 


\section{SATISFACTION WITHIN FAIRNESS AND BEHAVIOUR ABSTRACTION}

In this section we use behaviour abstraction [6; 7]. Before we define it this, we need a few preliminary results and definitions. First of all we will write $B \Vdash P$ for saying that $B$ satisfies $P$ within fairness:

$$
B \Vdash P \Longleftrightarrow \operatorname{pre}(B) \subseteq \operatorname{pre}(B \cap P) .
$$

Let $\mathcal{A}^{\infty}=\mathcal{A}^{*} \cup \mathcal{A}^{\omega}$. The leftquotient [3] of $M \subseteq \mathcal{A}^{\infty}$ by $w \in A^{*}$ is then the set

$$
\operatorname{cont}(w, M)=\left\{v \in \mathcal{A}^{\infty} \mid w v \in M\right\} .
$$

Lemma $4 \lim (L) \Vdash P$ if and only if

$$
\forall w \in \operatorname{pre}(\lim (L)): \exists x \in \operatorname{cont}(w, \lim (L)): w x \in P[7] .
$$

We can now give a definition of behaviour abstraction and explore its relation to satisfaction within fairness. It is defined by alphabetic language homomorphisms extended to $\omega$-languages [5]:

Definition $5 h: \mathcal{A}^{\infty} \rightarrow \mathcal{A}^{1 \infty}$ is an abstraction homomorphism if and only if the following conditions hold:

- $h(\mathcal{A}) \subseteq \mathcal{A}^{\prime} \cup\{\varepsilon\}$ (action renaming and hiding)

- $\forall v, w \in \mathcal{A}^{*}, x \in \mathcal{A}^{\omega}: h(v w)=h(v) h(w)$ and $h(v x)=h(v) h(x)$ (compatibility with concatenation)

- $h\left(\mathcal{A}^{\omega}\right) \subseteq \mathcal{A}^{\prime \omega}$ (undefined for $\omega$-words that would be taken to finite words).

Note that abstraction homomorphisms are partial mappings since they are not defined on $\omega$-words that would be taken to finitely long words. Also, note that they do not increase the length of words; that is, we always have that $|h(w)| \leq$ $|w|$. For an abstraction homomorphism $h$, we call $\lim (h(L))$ the abstraction of concrete behaviour $\lim (L)$. For prefix-closed regular $L$, $\lim (h(L))=$ $h(\lim (L))$ [7]. We will consider subsequently only behaviours that are the Eilenberg-limit of a prefix-closed regular language $L$ (i.e. $B=\lim (L), L=$ $\operatorname{pre}(L)$, and $L$ is regular). That means we are considering behaviours that can be represented by finite-state labelled transition systems.

In general, a property satisfied within fairness on the abstraction is not necessarily satisfied within fairness on the concrete behaviour in a corresponding way. We need an additional requirement to be satisfied by the abstraction homomorphism [8;9]: 
Essential information about properties that are satisfied within fairness by $\lim (L)$ is contained in the sets $\operatorname{cont}(w, L)$, for $w \in L$. At the abstract level, we obviously have access to $\operatorname{cont}(h(w), h(L))$, but we really need $h(\operatorname{cont}(w, L))$ in order to ensure that properties satisfied within fairness by the abstraction will also be satisfied within fairness by the concrete system in a corresponding way. Thus, we need to find conditions under which $\operatorname{cont}(h(w), h(L))$ can be used instead of $h(\operatorname{cont}(w, L))$.

In general, $h(\operatorname{cont}(w, L))$ is a proper subset of the set $\operatorname{cont}(h(w), h(L))$. In order to obtain sufficient information about $h(\operatorname{cont}(w, L))$ from considering $\operatorname{cont}(h(w), h(L))$, one would be tempted to require equality of the two sets. Those homomorphisms are continuation closed, since computation of the continuation (leftquotient) and the abstraction commute. However, this is stronger than needed. Indeed, since we are dealing with satisfaction within fairness, it is sufficient that the computations in $\operatorname{cont}(h(w), h(L))$ "eventually" become computations in $h(\operatorname{cont}(w, L))$ :

Definition $6 h$ is weakly continuation-closed on $L$ if and only if for all $w \in$ $A^{*}$, there exists $v \in \operatorname{cont}(h(w), h(L))$ such that cont $(v, \operatorname{cont}(h(w), h(L)))=$ $\operatorname{cont}(v, h(\operatorname{cont}(w, L)))$.

One should not worry about this rather technical definition. Its essence of this definition revealed by the following lemma:

Lemma 7 Let $L$ be regular. If $h(L)$ does not contain maximal words, ${ }^{2}$ then the condition

$$
\lim (h(L)) \Vdash P \text { if and only if } \lim (L) \Vdash h^{-1}(P)
$$

holds if and only if h is weakly continuation-closed on L [6].

Weakly continuation-closed homomorphisms are therefore precisely the class of homomorphisms that preserve satisfaction within fairness.

What is the essence of this result? To test whether the concrete behaviour $\lim (L)$ (i.e. the implementation's behaviour) satisfies property $h^{-1}(P)^{3}$ with the help of a safety approximation $S_{\lim (L) h^{-1}(P)}$ - a test that is practically impossible directly - we can test instead whether the abstract behaviour $\lim (h(L))$ satisfies abstract property $P$ with the help of $S_{\lim (h(L)) P}$ which can be constructed effectively. Since we can turn an observed (finite) behaviour of the system under test on-the-fly into its corresponding abstract behaviour by application of abstraction homomorphism $h$, we can then immediately test whether

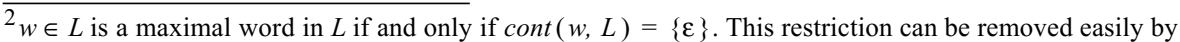
introducing additional "dummy" actions [6]. However, we avoid introducing additional notation here.

${ }^{3}$ Note that $h^{-1}(P)$ can even be a liveness property.
} 
the obtained trace is in $\operatorname{pre}\left(S_{\lim (h(L)) P}\right)$. If we tested the on-the-fly abstraction of the system traces exhaustively against $S_{\lim (h(L)) P}$ would immediately establish $\lim (L) \Vdash h^{-1}(P)$. We therefore have established an indirect way, with the help of abstraction, for testing even liveness properties of a system.

\section{CONCLUSIONS}

We have presented how liveness properties can be approximated by safety properties. The prefixes of these safety properties form then test cases that allow to check whether a system nearly meets some lieveness properties. "Nearly" means in this context that if the liveness property were not satisfied (wrt. lineartime verification), then it would only be detectable by observing the system for an infinite amount of time and would therefore be practically irrelevant.

Since constructing the safety property mentioned above requires construction of the intersection of the system implementation and the original property, the described approach is not yet feasible. However an abstraction result as presented can be used that allows to gain at least some benefit from the defined safety approximation. The abstraction result in the previous section requires the concrete behaviour (i.e. the implementation) to be representable by a finite-state labelled transition system and the abstraction to be weakly continuation-closed. These are two conditions that will not necessarily be satisfied in practice, or we may simply not know whether they are satisfied when testing the system. However, if the system is an implementation derived from some high-level specification where the high-level specification has a finite-state labelled transition system semantics, we can simply assume that the high-level specification is a weakly-continuation closed abstraction of the implementation and that the implementation itself also has a finite-state labelled transition system semantics. If the assumption is false, we will have lost information and our testing will inaccurate, even though it may seem to be exhaustive. We will still be able to spot some problems with respect to meeting progress properties that will not be testable without the presented approach.

The claim made is oviously quite vague, even though it is underpinned by interesting theoretical results, and needs solid experimental justification. Experiments using the concepts introduced in this paper will be part of our future work. We will therefore aspire co-operation with researchers who work on the practical application of system testing. Any comments and suggestions are welcome.

\section{REFERENCES}

[1] B. Alpern and F. B. Schneider. Defining liveness. Information Processing Letters, 21(4):181-185, October 1985. 
[2] B. Alpern and F. B. Schneider. Recognizing safety and liveness. Technical Report TR 86-727, Department of Computer Science, Cornell University, January 1986.

[3] S. Eilenberg. Automata, Languages and Machines, volume A. Academic Press, New York, 1974.

[4] J. L. Kelley. General Topology. Van Nostrand, Princeton, 1955.

[5] U. Nitsche. Propositional linear temporal logic and language homomorphisms. In A. Nerode and Y. V. Matiyasevich, editors, Proceedings of the 3rd International Symposium on Logical Foundations of Computer Science (LFCS'94), volume 813 of Lecture Notes in Computer Science, pages 265-277, Saint Petersburg, Russia, 1994. Springer Verlag.

[6] U. Nitsche and P. Ochsenschläger. Approximately satisfied properties of systems and simple language homomorphisms. Information Processing Letters, 60:201-206, 1996.

[7] U. Nitsche and P. Wolper. Relative liveness and behavior abstraction (extended abstract). In Proceedings of the 16th ACM Symposium on Principles of Distributed Computing (PODC'97), pages 45-52, Santa Barbara, CA, 1997.

[8] P. Ochsenschläger. Verifikation kooperierender Systeme mittels schlichter Homomorphismen. Arbeitspapiere der GMD 688, Gesellschaft für Mathematik und Datenverarbeitung (GMD), Darmstadt, Oktober 1992.

[9] P. Ochsenschäger. Verification of cooperating systems by simple homomorphisms using the product net machine. In J. Desel, A. Oberweis, and W. Reisig, editors, Workshop: Algorithmen und Werkzeuge für Petrinetze, pages 48-53. Humboldt Universität Berlin, 1994.

[10] A. Petrenko and N. Yevtushenko. On test derivation from partial specifications. In Proceedings of the IFIP TC6/WG6.1 International Conference on Formal Description Techniques for Distributed Systems and Communication Protocols and Protocol Specification, Testing and Verification (FORTE/PSTV'2000), pages 85-102, Pisa, Italy, October 2000. Springer Verlag.

[11] W. Thomas. Automata on infinite objects. In J. van Leeuwen, editor, Formal Models and Semantics, volume B of Handbook of Theoretical Computer Science, pages 133-191. Elsevier, 1990.

[12] U. Ultes-Nitsche. Satisfaction up to liveness - automatic verification based on exhaustive testing. In Proceedings of the IFIP TC6/WG6.1 International Conference on Formal Description Techniques for Distributed Systems and Communication Protocols and Protocol Specification, Testing and Verification (FORTE/ PSTV'2000), pages 237-248, Pisa, Italy, October 2000. Springer Verlag. 